\title{
CTNNB1 Mutations in Ovarian Microcystic Stromal Tumors: Identification of a Novel Deletion Mutation and the Use of Pyrosequencing to Identify Reported Point Mutation
}

\author{
KIYONG NA ${ }^{1}$, EUN KYUNG KIM ${ }^{1}$, WONJUN JANG ${ }^{2}$ and HYUN-SOO KIM ${ }^{1}$ \\ ${ }^{1}$ Department of Pathology, Severance Hospital, Yonsei University College of Medicine, Seoul, Republic of Korea; \\ ${ }^{2}$ Yonsei University College of Medicine, Seoul, Republic of Korea
}

\begin{abstract}
Background/Aim: Microcystic stromal tumor (MCST) is a rare stromal tumor of the ovary. In this study, we describe clinicopathological characteristics and results of mutational analyses of the CTNNB1gene in two cases of ovarian MCST and we provide a thorough review of previously published cases alongside our current cases and clarify the clinicopathological characteristics of ovarian MCST. Patients and Methods: Patients' age was 33 and 31 years, respectively. One patient presented with fever and low abdominal pain, whereas a pelvic mass was incidentally detected in another patient. Grossly, the cut surface of the tumors was mixed solid and cystic. Results: Histologically, the tumor characteristically displayed numerous microcysts, solid cellular areas, and intervening hyalinized stroma. Areas of moderate-to-severe nuclear pleomorphism with occasional multinucleated giant cells and bizarre nuclei were noted in one of the two cases. Immunohistochemically, both cases demonstrated diffuse and strong $\beta$-catenin expression in the nuclei and the cytoplasm. The tumor cells were also diffusely positive for CD10, vimentin, Wilms tumor 1, and cyclin D1. The tumor cells were consistently negative for $E$ cadherin, inhibin- $\alpha$, calretinin, estrogen receptor, and progesterone receptor. Mutational analyses using direct sequencing and pyrosequencing methods exhibited a single nucleotide mutation in CTNNB1 exon $3(c .122 C>T)$ in one case. We also found a novel deletion mutation in the same exon (c.88_99delTACCTGGACTCT) in another case. Conclusion: We demonstrated a previously reported CTNNB1 point-mutation using pyrosequencing and a novel
\end{abstract}

Correspondence to: Hyun-Soo Kim, Department of Pathology, Severance Hospital, Yonsei University College of Medicine, 50-1, Yonsei-ro, Seodaemun-gu, Seoul 03722, Republic of Korea. Tel: +82 222281794, +82 23620860, e-mail: hyunsookim@yuhs.ac

Key Words: Microcystic stromal tumor, ovary, $\beta$-catenin, CTNNB1, mutation, pyrosequencing. deletion mutation in ovarian MCSTs. The review of the literature of previously published cases in combination with our current cases clarifies the clinicopathological characteristics of ovarian MCST and the comprehensive analysis of these cases would expand our knowledge regarding ovarian MCST.

Microcystic stromal tumor (MCST) is a rare stromal tumor of the ovary. Since the first series of 16 cases published by Irving et al. (1), 17 additional cases have been reported to date (2-12). MCST is histologically characterized by a microcystic growth pattern, solid cellular areas, and intervening hyalinized stroma, accompanied by immunohistochemical evidence of sex cord-stromal differentiation. Maeda et al. (2) found that accumulation of nuclear $\beta$-catenin is relevant to the pathogenesis of MCST. More specifically, they observed two cases of ovarian MCST that exhibited a CTNNB1 point mutation in exon 3 and strong nuclear immunoreactivity for $\beta$-catenin.

$\beta$-catenin is a multifunctional protein involved in two independent processes- the transcriptional activation (6) and the cell-cell adhesion process (13). Cytoplasmic $\beta$-catenin is involved in Wnt signal transduction and activates transcription by translocating into the nucleus and forming a complex with T-cell factor, a transcription factor that upregulates cellular proliferation by increasing the transcription of $M Y C, C C N D 1$ and other genes $(6,14,15)$. Absence of Wnt signaling causes degradation of cytoplasmic $\beta$-catenin via a destruction complex consisting of APC, Axin, and glycogen synthase kinase-3 $\beta$, which leads to the phosphorylation and ubiquitination of $\beta$-catenin. Cells carrying mutations of either $A P C$ or CTNNB1 behave as if they are under continuous Wnt signaling and undergo dysregulated cellular proliferation $(6,16)$. Another role of $\beta$ catenin is participation in cell-to-cell interactions (contact inhibition) in close contact with cadherin, a molecule that links the extracellular molecules with the cytoskeleton (13). Cell-to-cell interactions, mediated by cadherins and catenins, 
result in the inhibition of cellular proliferation. Loss of contact inhibition due to mutations within the E-cadherin/ $\beta$ catenin axis is a characteristic of some carcinomas (13).

Recently, we detected a novel CTNNB1 deletion mutation in exon 3 in the ovarian MCST of a 33-year-old woman. This rare mutation has been reported in two cases of malignant hepatic tumors $(17,18)$, but not yet in ovarian MCST. Given our recent experience, we retrospectively reviewed our database and found an additional case of ovarian MCST harboring a heterozygous point mutation of CTNNB1. In this case, we employed pyrosequencing in addition to direct sequencing to confirm the presence of CTNNB1 mutation. This study also includes a comprehensive review of ovarian MCST clinicopathological characteristics based on previous case series and our own cases.

\section{Materials and Methods}

Case selection. The pathology database at Severance Hospital was queried for all cases that were diagnosed as MCST of the ovary between 2010 and 2015. A thorough search of the archival surgical pathology cases was performed using the key words "ovary", "stromal tumor" and "microcystic stromal tumor" Clinical and pathological information was obtained from electronic medical information systems and pathology reports. The clinical details that were reviewed included the age of patients at diagnosis, the clinical presentation, the associated medical conditions, the imaging findings, the serum tumor marker levels, the surgical and/or additional treatments and the current follow-up status. Pathological information included the location of tumor, the greatest dimension of tumor, the degree of nuclear atypia, the mitotic count, the presence of atypical mitotic figures and/or coagulative tumor cell necrosis and final the histopathological diagnosis. This study was reviewed and approved by the Institutional Review Board at Severance Hospital, Yonsei University Health System, Seoul, Republic of Korea (2016-1257-001).

Histopathological examination. The resection specimens were fixed in $10 \%$ neutral-buffered formalin and embedded in paraffin blocks. From each formalin-fixed, paraffin-embedded block, $4-\mu \mathrm{m}$ sections were cut and stained with hematoxylin and eosin. All available hematoxylin and eosin-stained slides were examined by routine light microscopy and the most representative block was chosen for immunohistochemical staining, direct sequencing and pyrosequencing.

Immunohistochemistry. The 4- $\mu \mathrm{m}$, formalin-fixed, paraffinembedded tissue sections were deparaffinized and rehydrated. Immunohistochemical staining was performed using the Ventana Benchmark XT automated staining system (Ventana Medical Systems, Inc., Tucson, AZ, USA) or Dako Omnis (Dako, Agilent Technologies, Inc., Carpinteria, CA, USA) according to the manufacturer's instructions. Antigen retrieval was performed using Cell Conditioning Solution (CC1; Ventana Medical Systems, Inc., Tucson, AZ, USA) or EnVision FLEX Target Retrieval Solution, High pH (Dako, Agilent Technologies, Inc., Carpinteria, CA, USA). The tissue sections were subsequently incubated with primary antibodies against $\beta$-catenin (1:200; clone 14, Cell Marque, Rocklin,
CA, USA), CD10 (1:50; clone 56C6, Novocastra, Leica Biosystems, Newcastle Upon Tyne, UK), vimentin (1:300; clone Vim384, Dako, Agilent Technologies, Inc., Carpinteria, CA, USA), Wilms tumor 1 (WT1; 1:200; clone 6F-H2, Cell Marque, Rocklin, CA, USA), cyclin D1 (1:100; clone SP4, Cell Marque, Rocklin, CA, USA), Ecadherin (1:100; clone NCH-38, Dako, Agilent Technologies, Inc., Carpinteria, CA, USA), inhibin- $\alpha$ (1:50; clone R1, Bio-Rad Laboratories, Hercules, CA, USA), calretinin (1:100; polyclonal, Cell Marque, Rocklin, CA, USA), estrogen receptor (ER; 1:150, clone 6F11, Novocastra, Leica Biosystems, Newcastle Upon Tyne, UK), progesterone receptor (PR; 1:100; clone 16, Novocastra, Leica Biosystems, Newcastle Upon Tyne, UK), androgen receptor (AR; 1:100; clone AR441, Dako, Agilent Technologies, Inc., Carpinteria, CA, USA), synaptophysin (1:900; clone DAK-SYNAP, Dako, Agilent Technologies, Inc., Carpinteria, CA, USA), chromogranin A (1:200; clone DAK-A3, Dako, Agilent Technologies, Inc., Carpinteria, CA, USA), CD56 (1:100; clone CD564, Novocastra, Leica Biosystems, Newcastle Upon Tyne, UK), CD99 (1:200; clone 12E7, Dako, Agilent Technologies, Inc., Carpinteria, CA, USA), Spalt-like protein 4 (SALL4; 1:100; clone 6E3, Cell Marque, Rocklin, CA, USA), smooth muscle actin (SMA; 1:500; clone 1A4, Dako, Agilent Technologies, Inc., Carpinteria, CA, USA), desmin (1:500; clone D33, Dako, Agilent Technologies, Inc., Carpinteria, CA, USA), CD31 (1:100; clone JC70A, Dako, Agilent Technologies, Inc., Carpinteria, CA, USA), CD34 (1:100; clone QBEnd 10, Dako, Agilent Technologies, Inc., Carpinteria, CA, USA) and Ki-67 (1:150; clone MIB-1, Dako, Agilent Technologies, Inc., Carpinteria, CA, USA). After chromogenic visualization using the ultraView Universal DAB Detection Kit (Ventana Medical Systems, Inc., Tucson, AZ, USA) or EnVision FLEX /HRP (Dako, Agilent Technologies, Inc., Carpinteria, CA, USA), all tissue slides were counterstained with hematoxylin, were dehydrated in graded alcohols and xylene and then embedded in mounting solution. Appropriate positive and negative controls were stained concurrently to validate the staining procedure. For positive controls, formalin-fixed and paraffin-embedded tissue blocks from normal human placenta, liver, ovary, and tonsil were stained. For negative controls, non-specific activity was assessed by omitting the primary antibodies.

Direct sequencing. Two cases of ovarian MCST were analyzed for CTNNB1 mutations, as previously described (2). Briefly, in both cases, histological confirmation for the presence of tumor tissue was performed by a Board-certified pathologist, who also marked the most dense tumor area on the hematoxylin and eosin section avoiding as much as possible necrotic and hemorrhagic areas. Tenmicrometer tissue sections were cut from formalin-fixed, paraffinembedded blocks containing MCST tissue. The marked areas were subsequently macrodissected with a scalpel and brought into 1.5 $\mathrm{ml}$ tubes for tissue digestion. Extraction of genomic DNAwas performed using a QIAamp DNA FFPE Tissue Kit (Qiagen, Valencia, CA, USA). DNA concentrations of extracted DNA were determined using spectrophotometer and adjusted to a concentration of $50 \mathrm{ng} / \mu \mathrm{l}$. Exon 3 of $C T N N B 1$ was amplified by polymerase chain reaction (PCR), using the following primer pairs: 5'-GATTTG ATGGAGTTGGACATGG-3' (sense) and 5'GCTACTTGTTCT TGAGTGAAGG-3' (antisense). Briefly, $50 \mathrm{ng}$ of DNA was amplified with the thermal cycling profile of $95^{\circ} \mathrm{C}$ for $30 \mathrm{~s}, 62^{\circ} \mathrm{C}$ for $45 \mathrm{~s}, 72^{\circ} \mathrm{C}$ for $45 \mathrm{~s}$, with a final extension at $72^{\circ} \mathrm{C}$ for $20 \mathrm{~min}$. PCR products were resolved by agarose gel 
electrophoresis to confirm successful amplification. The size of the target gene recovered was around $190 \mathrm{bp}$. Bidirectional Sanger sequencing of all PCR products was performed using a BigDye Terminator Cycle Sequencing Kit (Applied Biosystems, Foster City, CA, USA). Sequence analysis was performed using an Applied Biosystems 3130xl Genetic Analyzer (Applied Biosystems, Foster City, CA, USA).

Pyrosequencing. Based on our previous data (12), targeted analyses for CTNNB1 mutations at codons 33 and 41 within exon 3 were performed using pyrosequencing on a PyroMark Q24 instrument (Qiagen, Valencia, CA, USA)

Literature review. The Medline database was thoroughly searched using the PubMed retrieval service. Searches were performed in September 2016 using the key words "ovary," "ovarian," and "microcystic stromal tumor".

\section{Results}

Case presentation. Case 1: A 33-year-old multiparous woman was admitted to the Gynaecology Department for evaluation of a right pelvic mass incidentally detected by ultrasonography (US) during a routine checkup. Her medical history was unremarkable, except for a high body mass index (BMI; $30.12 \mathrm{~kg} / \mathrm{m}^{2}$ ). Physical examination revealed a nontender, barely palpable mass in the right pelvic area. Routine blood tests and serum levels of cancer antigen (CA) $125(13.7 \mathrm{U} / \mathrm{mL})$ and CA 19-9 $(5.2 \mathrm{U} / \mathrm{mL})$ were within normal range. Magnetic resonance imaging showed a relatively well-circumscribed, lobulated mass with solid and cystic components in the right ovary. She underwent a laparoscopic right salpingo-oophorectomy and partial omentectomy. She was discharged without complications and had no instances of tumor recurrence during the 57-month postoperative follow-up period.

Case 2: A 31-year-old nulliparous woman was admitted to the Gynaecology Department for fever and lower abdominal pain for 7 days. She was obese (BMI, $29.21 \mathrm{~kg} / \mathrm{m}^{2}$ ) and complained of metrorrhagia. Pelvic examination revealed a tender, palpable mass. Blood tests showed an elevated serum CA 125 level (125.0 U/mL) and a normal serum CA 19-9 level $(3.7 \mathrm{U} / \mathrm{mL})$. Magnetic resonance imaging revealed a huge mass with solid and cystic components in the left ovary. Positron emission tomography/computed tomography revealed increased 18-fluoro-2-deoxy-D-glucose uptake in several para-aortic lymph nodes, raising suspicion of a primary ovarian malignancy with lymph node metastases. She underwent left salpingo-oophorectomy with paraaortic lymph node sampling. Intraoperative inspection revealed a left ovarian mass with a fallopian tube twisted 360 degrees. She was discharged without complications and no tumor recurrence during a follow-up period of 20 months.

Histopathological and immunohistochemical findings. Grossly, all the ovarian masses exhibited smooth outer surfaces. They were well-circumscribed and displayed lobulated contours. They measured $8.6 \mathrm{~cm}$ (Case 1) and $24.0 \mathrm{~cm}$ (Case 2) at their greatest dimensions, respectively. The cut surface contained both solid (Figure 1A) and cystic areas. The solid portions of the masses were firm and tan-to-white. The cystic portions were inconsistently sized, with round-to-ovoid cysts separated by walls of variable thickness. The larger cysts contained brown serous fluid. The cystic wall was smooth and tan-togray, with some areas showing rough, granular surfaces. No grossly identifiable necrosis was observed.

Histologically, the tumor tissues consisted of microcysts, variable amounts of nested cellular regions and matrix. The microcysts showed distinctive architectural patterns and were predominantly composed of small, round-to-oval spaces. Areas of large, irregular spaces were often observed (Figure 1B). The cystic spaces were filled with clear or eosinophilic, acellular material. The solid portions were characterized by an admixture of irregularly distributed hypercellular and hypocellular areas (Figure 1C). The hypercellular areas consisted of solid sheets of tumor cells, numerous microcysts and intervening bundles of hyalinized fibrous bands. The hypocellular stroma commonly exhibited extensive hyalinization, myxoid degeneration, cystic change, which divided tumor cells into small nests. Most tumor cells were bland-looking with round-to-oval contours. Spindle-shaped cells in a streaming pattern were occasionally observed. The tumor cells possessed abundant, eosinophilic cytoplasm, with intracytoplasmic vacuoles. In one of the two cases (Case 1), the tumor cells exhibited minimal-to-mild nuclear atypia with uniform, small, round nuclear outlines, fine chromatin, and inconspicuous nucleoli (Figure 1D). In this case, mitotic activity was low (1 per 10 high-power fields) and without atypical mitotic figures. Coagulative tumor cell necrosis was absent. However, in the remaining case (Case 2), large areas of hemorrhage were seen (Figure 1E). The solid portions were hypercellular (Figure 1F). The tumor cells frequently showed large, hyperchromatic nuclei, and multinucleated giant cells with bizarre nuclei were also identified (Figure $1 \mathrm{G})$. Areas showing moderate-to-severe pleomorphism were located adjacent to areas of hemorrhage (Figure $1 \mathrm{H}$ ), inflammation, and granulation tissue. The mitotic rate $(3$ per 10 high-power fields) was slightly increased compared to the former case.

Immunohistochemically, both cases demonstrated diffuse and strong $\beta$-catenin expression in both the nuclei and the cytoplasm (Figure 1I and 1J). The tumor cells were also diffusely positive for CD10, vimentin, WT1, and cyclin D1 (Figure 1K). No significant increase in the Ki-67 labeling index was noted: approximately 2\% (Case 1) and 5\% (Case 2; Figure 1L), respectively. The tumor cells were consistently negative for E-cadherin, inhibin- $\alpha$, calretinin, ER, PR, AR, synaptophysin, chromogranin A, CD56, CD99, SALL4, SMA, desmin, CD31, and CD34. 


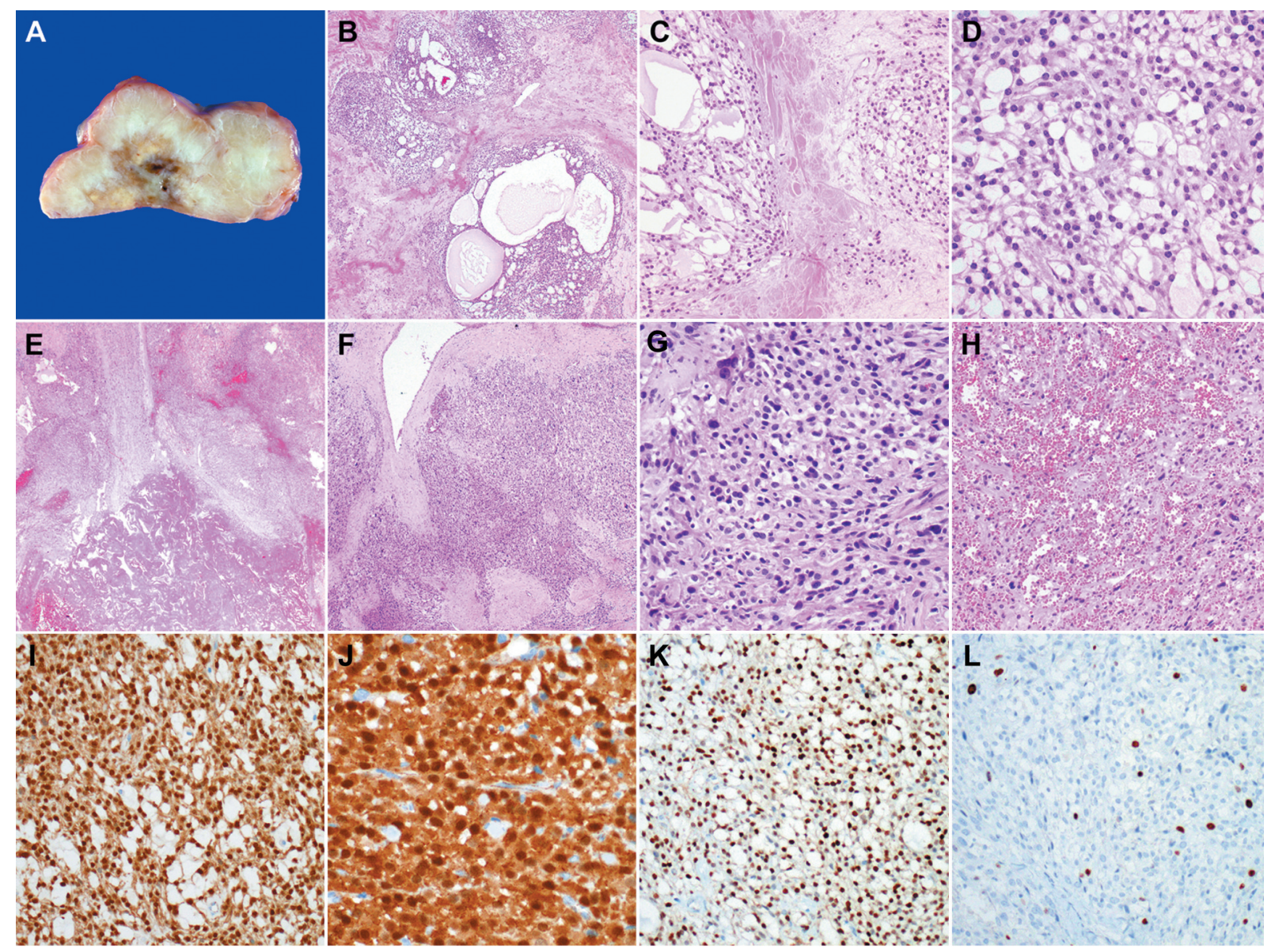

Figure 1. Pathological (A to H) and immunohistochemical (I to L) findings. Case 1. The mass is relatively well-circumscribed and lobulated. The solid portion of the tumor is firm and tan-to-white. A few hemorrhagic areas are noted (A). Low-power view reveals round-to-ovoid nests of tumor cells containing variable-sized, irregular-shaped cysts and hyalinized stroma (B). Medium-power view displays microcysts, aggregates of benignappearing tumor cells, and intervening hyalinized stroma $(C)$. High-power view reveals round, small, bland nuclei and clear-to-eosinophilic cytoplasm (D). Case 2. Scanning view shows large areas of hemorrhage and fibrin deposition (lower third) (E). Tumor cells showing a solid growth pattern are admixed with hyalinizing stroma $(F)$. Several areas exhibiting moderate-to-severe nuclear atypia $(G)$ are located adjacent to intratumoral hemorrhage (H). Immunostaining results. Tumor cell nuclei show diffuse and strong $\beta$-catenin (I and J) and cyclin D1 (K) immunoreactivity. The Ki-67 labeling index is low $(L)$.

Mutational analysis. Both cases were successfully sequenced for CTNNB1 mutations in exon 3 by direct sequencing. A single nucleotide mutation was detected in one case (Case 2), whereas deletion of 12 nucleotides was found in another case (Case 1). Case 2 exhibited a heterozygous missense mutation (c.122C $>$ T; Figure 2A) in CTNNB1 exon 3, leading to an amino acid replacement of threonine with isoleucine at codon 41 (p.T41I). Consistent with the results of direct sequencing, pyrosequencing revealed a point mutation (c.122C $>\mathrm{T}$ ) in exon 3 of CTNNB1 in Case 2 (Figure 2B). In contrast, sequencing analysis of Case 1 revealed a heterozygous deletion mutation of 12 consecutive nucleotides spanning from nucleotide position 88 to 99
(c.88_99delTACCTGGACTCT; Figure 2C). This mutation corresponded to a deletion of 4 consecutive amino acids from codons 30 to 33 (p.Y30_S33del).

Clinicopathological features. Table I summarizes the clinical features of 35 cases of MCST $(1-10,12)$. Patient age at the time of diagnosis ranged from 23 to 69 years (median $=44$ years). Twenty-one of the $35(60.0 \%)$ patients presented with a pelvic mass. The remaining patients complained of abdominal pain/discomfort or abnormal menstruation. Six of the $35(17.1 \%)$ patients had other neoplastic lesions in addition to ovarian MCST: numerous colonic adenomas associated with familial adenomatous polyposis $(2 / 6,33.3 \%)$, 
A
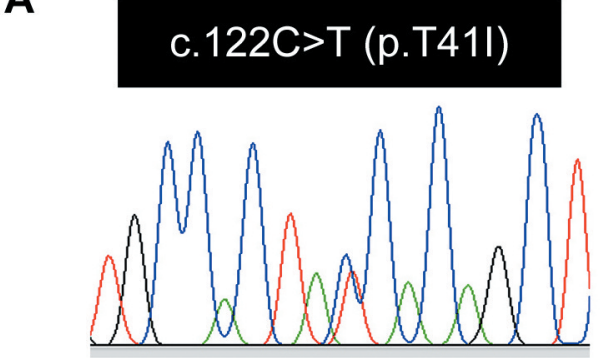

WT TGCCAC TACCACA G C T Mut
B

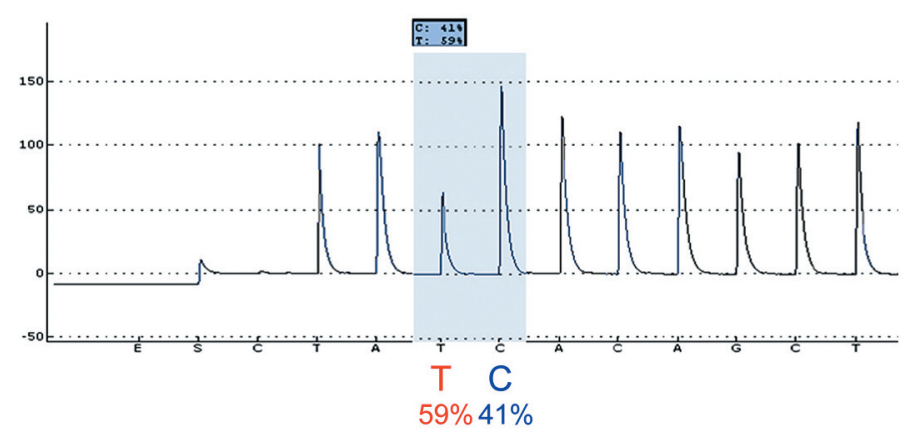

C

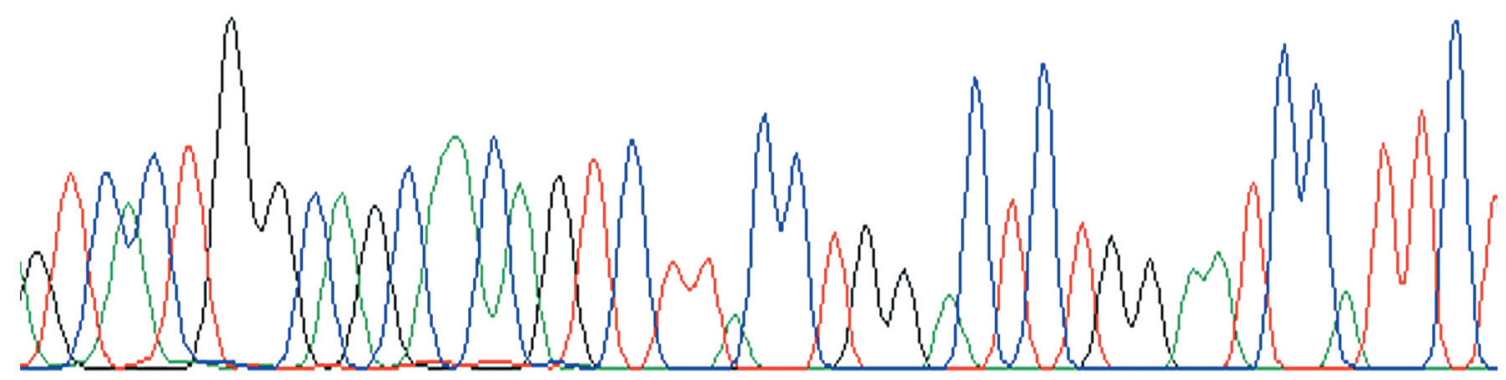

WT

CAC T G G CA G C AAC A G T C T TAC C T G G ACTCTG GAATC CA T T C

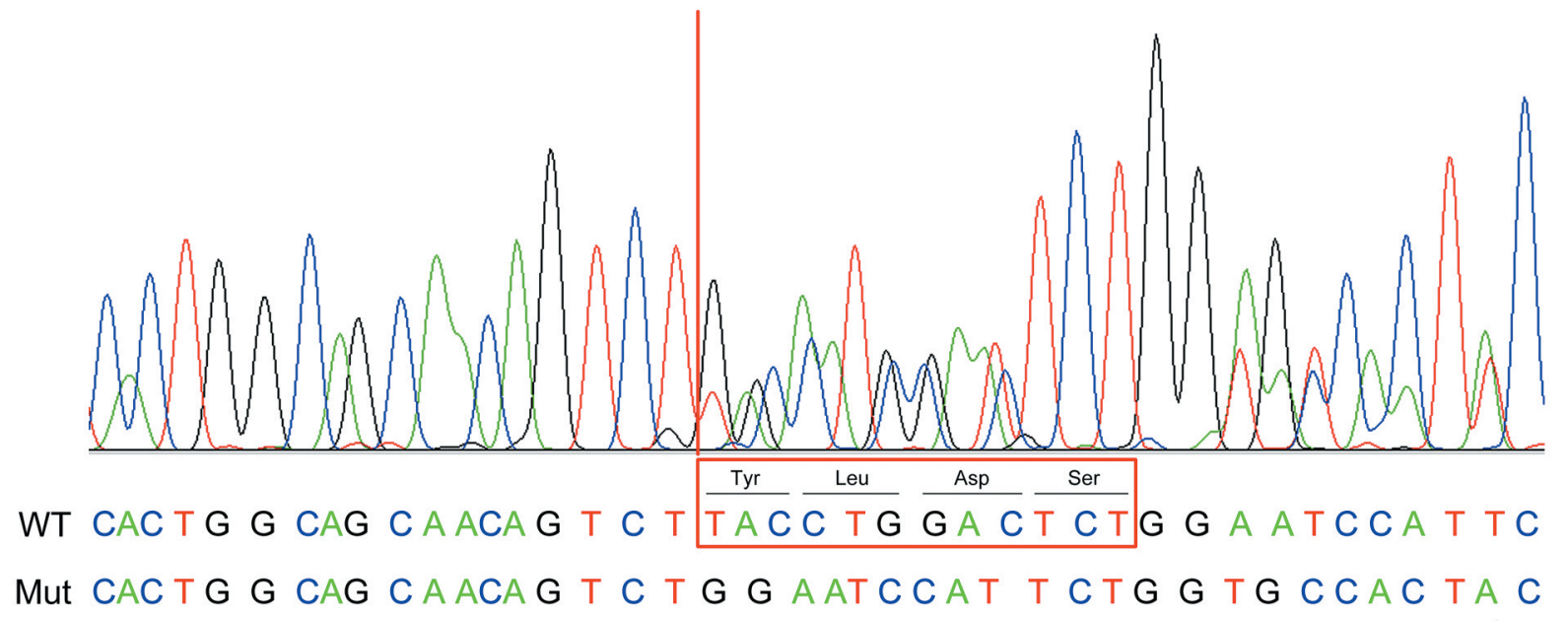

\section{c.88_99delTACCTGGACTCT (p.Y30_S33del)}

Figure 2. Results of Mutation Analysis. Sequence chromatograms and pyrograms showing point mutations in CTNNB1. In Case 1, a heterozygous missense mutation is detected in CTNNB1 exon 3 of codon 41 (c.122C>T; p.T41I) (A). Consistent with this result, pyrosequencing reveals that thiamine was aberrantly increased up to $59 \%$ at codon 41 (B). Sequence chromatogram displaying a novel deletion mutation in CTNNB1. Case 2 exhibits a heterozygous deletion mutation (c.88_99delTACCTGGACTCT). This mutation leads to the loss of 4 consecutive amino acids (p.Y30_S33del) (C).

endometrial carcinoma $(2 / 6,33.3 \%)$, ovarian solidpseudopapillary neoplasm $(1 / 6,16.7 \%)$, and ovarian mucinous cystadenoma $(1 / 6,16.7 \%)$. Radiologically, the tumors were mixed solid and cystic $(24 / 35,68.6 \%)$, multilocular cystic
$(5 / 35,14.3 \%)$, solid $(4 / 35,11.4 \%)$, or unilocular cystic $(2 / 35$, $5.7 \%)$. The serum level of CA 125 was evaluated in 15 $(42.9 \%)$ patients, of whom $3(20.0 \%)$ had elevated levels. More than half $(21 / 35,60.0 \%)$ of the patients underwent 
Table I. Clinical features.

\begin{tabular}{|c|c|c|c|c|c|c|c|c|c|c|c|c|}
\hline \multirow[t]{2}{*}{ Case } & \multirow[t]{2}{*}{$\begin{array}{c}\text { Year } \\
\text { reported }\end{array}$} & \multirow[t]{2}{*}{$\begin{array}{c}\text { Author } \\
\text { (reference) }\end{array}$} & \multirow[t]{2}{*}{$\begin{array}{l}\text { Age } \\
(\mathrm{yr})\end{array}$} & \multirow[t]{2}{*}{$\begin{array}{c}\text { Clinical } \\
\text { presentation }\end{array}$} & \multirow[t]{2}{*}{$\begin{array}{l}\text { Associated } \\
\text { condition }\end{array}$} & \multirow[t]{2}{*}{$\begin{array}{l}\text { Imaging } \\
\text { finding }\end{array}$} & \multicolumn{3}{|c|}{$\begin{array}{l}\text { Tumor } \\
\text { marker }\end{array}$} & \multirow[t]{2}{*}{$\begin{array}{l}\text { Surgery } \\
\text { status }\end{array}$} & \multirow[t]{2}{*}{$\begin{array}{c}\text { Current } \\
\text { (mo) }\end{array}$} & \multirow[t]{2}{*}{ FUT } \\
\hline & & & & & & & $\begin{array}{l}\text { CA } \\
125\end{array}$ & $\begin{array}{l}\text { CA } \\
19-9\end{array}$ & CEA & & & \\
\hline 1 & 2017 & $\begin{array}{l}\text { Na et al. } \\
\text { (this study) }\end{array}$ & 33 & Incidental & Obesity & Solid-cystic & 13.7 & 5.2 & NA & $\mathrm{RSO}+\mathrm{OM}$ & NED & 57 \\
\hline 2 & & & 31 & $\begin{array}{l}\text { LAP, fever, } \\
\text { metrorrhagia }\end{array}$ & Obesity & Solid-cystic & 125.0 & 3.7 & NA & LSO+PALNS & NED & 20 \\
\hline 3 & 2016 & $\begin{array}{l}\text { Lee } e t a l . \\
\quad(12)\end{array}$ & 24 & $\begin{array}{l}\text { Abdominal } \\
\text { discomfort }\end{array}$ & Obesity, DM & Multilocular cystic & 19.0 & 6.4 & NA & LSO & NED & 25 \\
\hline 4 & & & 31 & Pelvic mass & None & Solid-cystic & 205.3 & NA & NA & $\begin{array}{l}\text { LSO+PALNS+ } \\
\text { Peritonectomy }\end{array}$ & NED & 3 \\
\hline 5 & 2016 & Liu et al. (10) & 23 & Menorrhagia & FAP & Solid-cystic & NA & NA & NA & $\mathrm{TH}+\mathrm{RSO}+\mathrm{LS}$ & NA & NA \\
\hline 6 & 2015 & Kang et al. (9) & 41 & $\begin{array}{l}\text { Abdominal } \\
\text { discomfort }\end{array}$ & None & Solid & WNL & WNL & $\mathrm{NA}$ & LSO & NA & NA \\
\hline 7 & 2015 & $\begin{array}{l}\text { Podduturi } \\
\text { et al. (8) }\end{array}$ & 50 & $\begin{array}{l}\text { Abdominal } \\
\text { discomfort }\end{array}$ & None & Multilocular cystic & 41.0 & NA & WNL & $\begin{array}{l}\mathrm{TH}+\mathrm{BSO}+ \\
\mathrm{LND}+\mathrm{OM}\end{array}$ & NA & NA \\
\hline 8 & 2015 & Gunes et al. (7) & 52 & Pelvic mass & Ovarian MCA & Solid-cystic & NA & NA & NA & $\mathrm{TH}+\mathrm{BSO}+\mathrm{OM}$ & NED & 3 \\
\hline 9 & 2015 & Chen et al. (6) & 47 & Pelvic mass & Ovarian SPN & Solid-cystic & WNL & WNL & WNL & LSO & NED & 18 \\
\hline 10 & 2015 & Bi et al. (5) & 69 & Pelvic mass & None & Solid-cystic & WNL & WNL & NA & LSO & NED & 60 \\
\hline 11 & & & 29 & Pelvic mass & None & Solid-cystic & WNL & WNL & NA & LO & NED & 18 \\
\hline 12 & & & 40 & Pelvic mass & None & Solid-cystic & WNL & WNL & NA & LO & NED & 7 \\
\hline 13 & & & 65 & Pelvic mass & None & Multilocular cystic & WNL & WNL & NA & $\mathrm{TH}+\mathrm{BSO}$ & & NA \\
\hline 14 & & & 57 & Pelvic mass & None & Unilocular cystic & WNL & WNL & NA & $\mathrm{TH}+\mathrm{BSO}$ & NED & 59 \\
\hline 15 & & & 41 & $\begin{array}{l}\text { Pelvic mass, } \\
\text { oligomenorrhea }\end{array}$ & None & Solid-cystic & WNL & WNL & $\mathrm{NA}$ & $\begin{array}{c}\mathrm{TH}+\mathrm{BSO}+ \\
\mathrm{OM}\end{array}$ & NED & 2 \\
\hline 16 & 2015 & Lee et al. (4) & 40 & $\begin{array}{l}\text { Abdominal } \\
\text { discomfort }\end{array}$ & FAP & Solid-cystic & WNL & WNL & WNL & LO & NED & 9 \\
\hline 17 & 2014 & Yang et al. (3) & 45 & $\begin{array}{l}\text { Abdominal } \\
\text { discomfort }\end{array}$ & None & Solid-cystic & 32.6 & NA & WNL & LO & NA & NA \\
\hline 18 & 2011 & Maeda & 33 & Pelvic mass & None & Solid-cystic & WNL & WNL & NA & $\mathrm{RSO}+\mathrm{OM}$ & NED & 14 \\
\hline 19 & & et al. (12) & 41 & $\begin{array}{l}\text { Abdominal } \\
\text { discomfort }\end{array}$ & None & $\begin{array}{l}\text { Multilocular } \\
\text { cystic }\end{array}$ & NA & NA & NA & RSO & NED & 4 \\
\hline 20 & 2009 & $\begin{array}{l}\text { Irving } \\
\text { et al. (1) }\end{array}$ & 62 & Pelvic mass & $\begin{array}{c}\text { EM cancer, } \\
\text { ovarian } \\
\text { hyperthecosis }\end{array}$ & Solid-cystic & NA & NA & NA & $\begin{array}{l}\text { TH+BSO+ } \\
\text { LND+OM }\end{array}$ & NA & NA \\
\hline 21 & & & 45 & $\begin{array}{l}\text { Abdominal } \\
\text { discomfort }\end{array}$ & None & Solid-cystic & NA & NA & NA & $\mathrm{TH}+\mathrm{BSO}$ & NA & NA \\
\hline 22 & & & 51 & Pelvic mass & None & Solid-cystic & NA & NA & NA & $\mathrm{TH}+\mathrm{BSO}+\mathrm{OM}$ & NA & NA \\
\hline 23 & & & 29 & Incidental & None & Multilocular cystic & NA & NA & NA & LO & NA & NA \\
\hline 24 & & & 58 & Pelvic mass & None & Unilocular cystic & NA & NA & NA & $\mathrm{TH}+\mathrm{BSO}+\mathrm{LND}$ & NA & NA \\
\hline 25 & & & 26 & Abdominal pain & None & Solid-cystic & NA & NA & NA & BSO & NA & NA \\
\hline 26 & & & 29 & Pelvic mass & None & Solid-cystic & NA & NA & NA & RO & NA & NA \\
\hline 27 & & & 45 & $\begin{array}{l}\text { Pelvic mass, } \\
\text { menorrhagia }\end{array}$ & None & Solid & NA & NA & NA & LSO & NA & NA \\
\hline 28 & & & 63 & Pelvic mass & None & Solid-cystic & NA & NA & NA & RO & NA & NA \\
\hline 29 & & & 56 & Pelvic mass & None & Solid-cystic & NA & NA & NA & BSO & NED & 18 \\
\hline 30 & & & 45 & Incidental & None & Solid-cystic & NA & NA & NA & $\mathrm{TH}+\mathrm{BSO}$ & NED & 34 \\
\hline 31 & & & 55 & Pelvic mass & None & Solid-cystic & NA & NA & NA & $\mathrm{TH}+\mathrm{BSO}$ & NED & 50 \\
\hline 32 & & & 44 & Pelvic mass & None & Solid-cystic & NA & NA & NA & $\mathrm{TH}+\mathrm{BSO}$ & NED & 51 \\
\hline 33 & & & 36 & Pelvic mass & None & Solid-cystic & NA & NA & NA & LSO & NED & 52 \\
\hline 34 & & & 37 & $\begin{array}{c}\text { Dysfunctional } \\
\text { uterine } \\
\text { bleeding }\end{array}$ & $\begin{array}{c}\text { EM cancer, } \\
\text { ovarian } \\
\text { hyperthecosis }\end{array}$ & Solid & NA & NA & NA & $\mathrm{TH}+\mathrm{BSO}$ & NED & 53 \\
\hline 35 & & & 39 & Pelvic mass & None & Solid & NA & NA & NA & RSO & NED & 150 \\
\hline
\end{tabular}

BSO: Bilateral salpingo-oophorectomy; CA 125: cancer antigen 125; EM: endometrium; FAP: familial adenomatous polyposis; FUT: follow-up time; LAP: low abdominal pain; MCA: mucinous cystadenoma; LND: lymph node dissection; LO: left oophorectomy; LSO: left salpingooophorectomy; NA: not applicable; NED: no evidence of disease; OM: omentectomy; PALNS: paraaortic lymph node sampling; RO: right oophorectomy; RSO: right salpingo-oophorectomy; SPN: solid-pseudopapillary neoplasm; TH: total hysterectomy; WNL: within normal limit. 
Table II. Pathological features.

\begin{tabular}{|c|c|c|c|c|c|c|c|c|}
\hline \multirow[t]{2}{*}{ Case } & \multirow{2}{*}{$\begin{array}{c}\text { Year } \\
\text { reported }\end{array}$} & \multirow{2}{*}{$\begin{array}{c}\text { Author } \\
\text { (reference) }\end{array}$} & \multirow{2}{*}{$\begin{array}{c}\text { Tumor } \\
\text { location }\end{array}$} & \multirow{2}{*}{$\begin{array}{c}\text { Tumor } \\
\text { size }(\mathrm{cm})\end{array}$} & \multicolumn{4}{|c|}{ Histopathological feature } \\
\hline & & & & & Mitotic count & Atypical mitotic figure & MNGC and bizarre nuclei & CTCN \\
\hline 1 & 2017 & $\mathrm{Na}$ et al. & RO & 8.6 & 1/10 HPFs & None & None & None \\
\hline 2 & & (this study) & LO & 24.0 & 3/10 HPFs & None & Occasional & Multifocal \\
\hline 3 & 2016 & Lee et al. (12) & LO & 18.0 & 3/10 HPFs & None & Occasional & $\begin{array}{l}\text { Multifocal; } \\
\text { microscopic }\end{array}$ \\
\hline 4 & & & LO & 20.0 & 0/10 HPFs & None & None & None \\
\hline 5 & 2016 & Liu et al. (10) & RO & 16.0 & $<1 / 50$ HPFs & None & None & None \\
\hline 6 & 2015 & Kang et al. (9) & LO & 7.8 & 1/10 HPFs & None & None & None \\
\hline 7 & 2015 & Podduturi et al. (8) & RO & 14.0 & None & None & None & None \\
\hline 8 & 2015 & Gunes et al. (7) & RO & 7.0 & 1/10 HPFs & None & Up to $30 \%$ & Focal \\
\hline 9 & 2015 & Chen et al. (6) & LO & 6.0 & $<1 / 10$ HPFs & None & $<1 \%$ & None \\
\hline 10 & 2015 & Bi et al. (5) & LO & 15 & $0-2$ & None & Occasional & None \\
\hline 11 & & & LO & 5.5 & $0-2$ & None & Occasional & None \\
\hline 12 & & & LO & 8.0 & $0-2$ & None & Occasional & None \\
\hline 13 & & & LO & 11.0 & $0-2$ & None & Occasional & None \\
\hline 14 & & & LO & 10.0 & $0-2$ & None & Occasional & None \\
\hline 15 & & & LO & 7.0 & $0-2$ & None & Occasional & None \\
\hline 16 & 2015 & Lee et al. (4) & $\mathrm{LO}$ & 15.0 & $0-1$ & None & None & Multifocal \\
\hline 17 & 2014 & Yang et al. (3) & LO & 18.0 & None & None & Focal & Focal \\
\hline 18 & 2011 & Maeda et al. (2) & RO & 11.5 & None & None & None & None \\
\hline 19 & & & $\mathrm{RO}$ & 9.5 & None & None & Focal & None \\
\hline 20 & 2009 & Irving et al. (1) & LO & 27.0 & 1 & None & Focal & Focal \\
\hline 21 & & & LO & 10.0 & 1 & None & Focal & None \\
\hline 22 & & & LO & 12.0 & 1 & None & Focal & None \\
\hline 23 & & & LO & 10.0 & 1 & None & Focal & None \\
\hline 24 & & & RO & 6.2 & 1 & None & Focal & None \\
\hline 25 & & & NA & 8.5 & 1 & None & Focal & None \\
\hline 26 & & & RO & 6.0 & 1 & None & Focal & Focal \\
\hline 27 & & & LO & 4.0 & 1 & None & Focal & None \\
\hline 28 & & & RO & 4.6 & 1 & None & Focal & None \\
\hline 29 & & & NA & 4.2 & 0 & None & Focal & None \\
\hline 30 & & & RO & 4.5 & 0 & None & None & None \\
\hline 31 & & & LO & 24.0 & 0 & None & None & None \\
\hline 32 & & & LO & 7.0 & 0 & None & None & None \\
\hline 33 & & & LO & 3.0 & 0 & None & None & None \\
\hline 34 & & & RO & 2.0 & 2 & None & None & None \\
\hline 35 & & & $\mathrm{RO}$ & 6.4 & 2 & None & None & None \\
\hline
\end{tabular}

CTCN: Coagulative tumor cell necrosis HPFs: high-power fields; LO: left ovary; MNGC: multinucleated giant cells; NA: not applicable; RO: right ovary.

oophorectomy with or without salpingectomy, and the remaining patients $(14 / 35,40.0 \%)$ underwent total hysterectomy and salpingo-oophorectomy with or without omentectomy. Data for clinical follow-up were available for $21(60.0 \%)$ patients. The follow-up period ranged from 2 to 150 months. None of the patients experienced recurrent disease after treatment. Although the prognosis of ovarian MCST needs to be elucidated, a lack of recurrent cases suggests the benign nature of this tumor.

Table II summarizes pathological features of the 35 MCST cases $(1-10,12)$. The laterality of ovarian involvement was described in 33 of 35 patients $(94.3 \%)$ and the left ovary
$(21 / 33,63.6 \%)$ was more frequently involved than the right ovary $(12 / 33,36.4 \%)$. The sizes of the tumors ranged from 2 to $27 \mathrm{~cm}$ (mean, $10.3 \mathrm{~cm}$ ). MCST histological features were characterized by the following: well-circumscribed border; admixture of irregularly distributed microcysts, macrocysts, and solid sheets of tumor cells; intervening thick fibrous stroma with varying degrees of hyalinization; monotonous tumor cells with bland, round-to-ovoid nuclei; and areas of hemorrhage. In addition to these findings, tumor necrosis was observed in $7(20.0 \%)$ cases and multinucleated giant cells and bizarre nuclei were present in $21(60.0 \%)$ cases. However, these histological findings do not seem to 
Table III. Summary of reported CTNNB1 or APC mutations in patients with ovarian MCST.

\begin{tabular}{|c|c|c|c|c|c|c|}
\hline \multirow{2}{*}{$\begin{array}{l}\text { Year reported (reference) } \\
2017 \text { (this study) }\end{array}$} & \multirow{2}{*}{$\frac{\text { Number of case }}{1}$} & \multicolumn{2}{|c|}{ Location } & \multirow{2}{*}{$\begin{array}{c}\text { Type } \\
\begin{array}{c}\text { Heterozygous deletion } \\
\text { mutation }\end{array}\end{array}$} & \multirow{2}{*}{$\frac{\text { Nucleotide }}{\text { c.88_99del12 }}$} & \multirow{2}{*}{$\frac{\text { Amino acid }}{\text { p.Y30_S33del }}$} \\
\hline & & CTNNB1 & Exon 3 & & & \\
\hline $2016(12)$ & 2 & CTNNB1 & Exon 3 & Heterozygous missense & c. $.98 \mathrm{C}>\mathrm{G}$ & p.S33C \\
\hline $2011(2)$ & 2 & & & mutation & & \\
\hline 2017 (this study) & 1 & CTNNB1 & Exon 3 & Heterozygous missense & c. $122 \mathrm{C}>\mathrm{T}$ & pT41I \\
\hline $2015(5)$ & 1 & & & mutation & & \\
\hline 2015 (11) & 2 & CTNNB1 & Exon 3 & Heterozygous missense & c. $101 \mathrm{G}>\mathrm{A}$ & p.G34E \\
\hline $2015(8)$ & 1 & & & mutation & & \\
\hline $2015(5)$ & 1 & & & & & \\
\hline $2015(9)$ & 1 & CTNNB1 & Exon 3 & Heterozygous missense & c. $97 \mathrm{~T}>\mathrm{C}$ & p.S33P \\
\hline 2015 (5) & 1 & & & mutation & & \\
\hline 2015 (11) & 1 & CTNNB1 & Exon 3 & Heterozygous missense & c. $110 \mathrm{C}>\mathrm{G}$ & p.S37C \\
\hline $2015(5)$ & 1 & & & mutation & & \\
\hline 2015 (7) & 1 & CTNNB1 & Exon 3 & $\begin{array}{l}\text { Heterozygous missense } \\
\text { mutation }\end{array}$ & c. $110 \mathrm{C}>\mathrm{A}$ & p.S37Y \\
\hline 2015 (11) & 4 & CTNNB1 & Exon 3 & $\begin{array}{l}\text { Heterozygous missense } \\
\text { mutation }\end{array}$ & c. $.95 \mathrm{~A}>\mathrm{T}$ & p.D32V \\
\hline $2015(11)$ & 1 & CTNNB1 & Exon 3 & $\begin{array}{l}\text { Heterozygous missense } \\
\text { mutation }\end{array}$ & c. $104 \mathrm{~T}>\mathrm{G}$ & p.I35S \\
\hline $2015(11)$ & 1 & CTNNB1 & Exon 3 & $\begin{array}{l}\text { Heterozygous missense } \\
\text { mutation }\end{array}$ & c. $109 \mathrm{~T}>\mathrm{C}$ & p.S37P \\
\hline 2015 (4) & 1 & $A P C^{*}$ & Exon 11 & $\begin{array}{c}\text { Heterozygous deletion } \\
\text { mutation }\end{array}$ & c.1540_1540delG & p.A514fs*9 \\
\hline $2016(10)$ & 1 & $A P C^{*}$ & Intron 6 & Splice site mutation & c. $730-1 \mathrm{G}>\mathrm{T}$ & Unknown \\
\hline
\end{tabular}

*Detected in patients with familial adenomatous polyposis.

reflect aggressive behavior; tumor recurrence was absent in all cases after surgery. Since one case presented with focal moderate-to-severe nuclear pleomorphism, multinucleated giant cells and bizarre nuclei with adjacent intratumoral hemorrhage, the nature of these histological findings are speculated to be reactive rather than neoplastic. Mitotic figures were identified in up to 3 per 10 high power fields, but none of the tumors showed atypical mitotic figures.

\section{Discussion}

MCST pathogenesis is linked to $\beta$-catenin stabilization involving $\beta$-catenin/Wnt signaling. Recent studies have shown that CTNNB1 mutations are highly prevalent in MCST cases $(2,5,7-9,11,12)$. Previously reported cases exhibited singlenucleotide point mutations in CTNNB1 exon 3 at codons 32, 33, 34, 35, 37, and 41 (Table III). These mutations can affect glycogen synthase kinase- $3 \beta$ phosphorylation sites and disturb the degradation of $\beta$-catenin. In this study, we demonstrated for the first time the presence of a deletion mutation in CTNNB1 exon 3 in ovarian MCST (c.88_99delTACCTGGACTCT; p.Y30_S33del). Mutations of CTNNB1 other than point mutations have not yet been reported in MCST. Two previous studies reported an identical deletion mutation in hepatocellular carcinoma and hepatoblastoma $(17,18)$. Since the deletion mutation affects codons 30, 31, 32 and 33 in CTNNB1 exon 3, this mutation is considered to have a similar role to that of the point mutations identified in MCST. Other mutations reported in MCST include $A P C$ deletion and point mutations in two patients with familial adenomatous polyposis $(4,10)$. Because APC is an important molecule in the regulation of $\beta$-catenin turnover, an $A P C$ mutation would also play a crucial role in the pathogenesis of MCST through $\beta$-catenin stabilization. CTNNB1 mutation in MCST is highly correlated with immunohistochemical detection of nuclear $\beta$-catenin. Only 6 cases showing nuclear $\beta$-catenin immunoreactivity exhibited wild-type $C T N N B 1(5,19)$. In contrast to the association between $C T N N B 1$ mutation and $\beta$-catenin, FOXL2 mutations were not detected in MCST cases showing FOXL2 expression (19). Although the mechanism for increased FOXL2 expression in MCST remains unclear, based on the fact that FOXL2 mutation is crucial for adult granulosa cell tumor pathogenicity (19), the molecular pathogenesis of MCST is presumed to be different from that of adult granulosa cell tumor.

Irving et al. (11) investigated the immunohistochemical expression of various markers in 15 cases of MCST, including 11 cases documented in their previous report in 2009 (1). We reviewed the immunohistochemical findings of these cases, as well as 17 previously published cases $(2-10,12)$ and our two cases described earlier. Table IV summarizes the 
Table IV. Immunostaining results.

\begin{tabular}{|c|c|c|c|c|c|c|c|c|c|c|c|c|c|c|c|c|c|c|c|}
\hline Case & $\begin{array}{l}\text { Year } \\
\text { reported }\end{array}$ & $\begin{array}{c}\text { Author } \\
\text { (reference) }\end{array}$ & $\beta$ cat & Ecad & CD10 & VIM & WT1 & $\begin{array}{l}\text { Cyclin } \\
\text { D1 }\end{array}$ & Ki-67 & INH & CRT & $\begin{array}{l}\text { ER, } \\
\text { PR }\end{array}$ & $\begin{array}{l}\mathrm{SNP} \\
\mathrm{CgA}\end{array}$ & CD56 & CD99 & $\begin{array}{l}\text { CK, } \\
\text { EMA }\end{array}$ & CD117 & $\begin{array}{l}\text { CD31, } \\
\text { CD34 }\end{array}$ & $\begin{array}{l}\text { SMA, } \\
\text { desmin }\end{array}$ \\
\hline $\begin{array}{l}1 \\
2\end{array}$ & 2017 & $\begin{array}{l}\text { Na et al. } \\
\text { (this study) }\end{array}$ & $2 / 2$ & $0 / 2$ & $2 / 2$ & $2 / 2$ & $2 / 2$ & $2 / 2$ & $\begin{array}{l}2 \% \\
3 \%\end{array}$ & $0 / 2$ & $0 / 2$ & $\begin{array}{l}0 / 2 \\
0 / 2\end{array}$ & $\begin{array}{l}0 / 2 \\
0 / 2\end{array}$ & $0 / 2$ & $0 / 2$ & $\begin{array}{l}0 / 2 \\
0 / 2\end{array}$ & $0 / 2$ & $\begin{array}{l}0 / 2 \\
0 / 2\end{array}$ & $\begin{array}{l}0 / 2 \\
0 / 2\end{array}$ \\
\hline $3-4$ & 2016 & $\begin{array}{l}\text { Lee } \text { et al. } \\
\quad(12)\end{array}$ & $2 / 2$ & NA & $2 / 2$ & $2 / 2$ & NA & NA & NA & NA & NA & NA & NA & NA & $1 / 2$ & NA & NA & NA & NA \\
\hline 5 & 2016 & $\begin{array}{l}\text { Liu et al. } \\
\quad(10)\end{array}$ & $1 / 1$ & NA & $1 / 1$ & $1 / 1$ & $1 / 1$ & $1 / 1$ & NA & $0 / 1$ & $0 / 1$ & NA & $\begin{array}{c}0 / 1 \\
0 / 1\end{array}$ & $0 / 1$ & NA & $\begin{array}{l}0 / 1 \\
\text { NA }\end{array}$ & NA & NA & NA \\
\hline 6 & 2015 & $\begin{array}{c}\text { Kang et al. } \\
\text { (9) }\end{array}$ & $1 / 1$ & 0/1 & $1 / 1$ & $1 / 1$ & $1 / 1$ & NA & $0 \%$ & 0/1 & $0 / 1$ & $\begin{array}{l}0 / 1 \\
0 / 1\end{array}$ & NA & NA & NA & NA & NA & NA & NA \\
\hline 7 & 2015 & $\begin{array}{l}\text { Podduturi } \\
\text { et al. (8) }\end{array}$ & $1 / 1$ & NA & $1 / 1$ & $1 / 1$ & $1 / 1$ & NA & NA & $1 / 1$ & $1 / 1$ & NA & NA & NA & NA & $\begin{array}{c}0 / 1 \\
1 / 1\end{array}$ & $1 / 1$ & NA & NA \\
\hline 8 & 2015 & $\begin{array}{l}\text { Gunes } \\
\text { et al. (7) }\end{array}$ & $1 / 1$ & NA & $1 / 1$ & $1 / 1$ & $1 / 1$ & NA & $16 \%$ & 0/1 & $0 / 1$ & NA & $\begin{array}{l}0 / 1 \\
0 / 1\end{array}$ & $0 / 1$ & NA & $\begin{array}{l}1 / 1 \\
0 / 1\end{array}$ & NA & NA & NA \\
\hline 9 & 2015 & $\begin{array}{l}\text { Chen et al. } \\
\text { (5) }\end{array}$ & $1 / 1$ & NA & $1 / 1$ & $1 / 1$ & $1 / 1$ & NA & $5 \%$ & $0 / 1$ & $0 / 1$ & $\begin{array}{c}\text { NA, } \\
1 / 1\end{array}$ & $\begin{array}{l}0 / 1 \\
0 / 1\end{array}$ & $1 / 1$ & $0 / 1$ & $\begin{array}{l}1 / 1 \\
0 / 1\end{array}$ & NA & NA & $\begin{array}{l}0 / 1 \\
0 / 1\end{array}$ \\
\hline $10-15$ & 2015 & $\begin{array}{l}\text { Bi et al. } \\
\text { (4) }\end{array}$ & $5 / 6$ & $0 / 5$ & $6 / 6$ & $6 / 6$ & $6 / 6$ & NA & NA & $0 / 6$ & $0 / 6$ & $\begin{array}{l}\text { NA, } \\
2 / 5\end{array}$ & NA & $1 / 5$ & $3 / 5$ & NA & $0 / 5$ & NA & NA \\
\hline 16 & 2015 & $\begin{array}{l}\text { Lee } \text { et al. } \\
\text { (3) }\end{array}$ & $1 / 1$ & NA & $1 / 1$ & $1 / 1$ & NA & NA & $<1 \%$ & $0 / 1$ & $0 / 1$ & $\begin{array}{l}0 / 1 \\
0 / 1\end{array}$ & NA & NA & NA & $\begin{array}{l}0 / 1 \\
0 / 1\end{array}$ & $0 / 1$ & NA & NA \\
\hline 17 & 2014 & $\begin{array}{l}\text { Yang et al. } \\
\text { (2) }\end{array}$ & $1 / 1$ & NA & $1 / 1$ & $1 / 1$ & NA & NA & $<10 \%$ & $0 / 1$ & $1 / 1$ & NA & NA & NA & NA & $\begin{array}{l}0 / 1 \\
0 / 1\end{array}$ & NA & NA & NA \\
\hline $\begin{array}{l}18 \\
19\end{array}$ & 2011 & $\begin{array}{c}\text { Maeda } \\
\text { et al. }(10)\end{array}$ & $2 / 2$ & NA & $2 / 2$ & $2 / 2$ & $2 / 2$ & NA & $\begin{array}{c}7 \% \\
10 \%\end{array}$ & $0 / 2$ & $0 / 2$ & $\begin{array}{l}0 / 2 \\
0 / 2\end{array}$ & $\begin{array}{l}0 / 2 \\
0 / 2\end{array}$ & $0 / 2$ & $0 / 2$ & $\begin{array}{l}1 / 2 \\
0 / 2\end{array}$ & NA & $\begin{array}{l}0 / 2, \\
0 / 2\end{array}$ & $\begin{array}{l}0 / 2 \\
0 / 2\end{array}$ \\
\hline 20-34 & 2015 & $\begin{array}{c}\text { Irving } \\
\text { et al. (11) }\end{array}$ & $15 / 15$ & $0 / 15$ & $15 / 15$ & $15 / 15$ & $15 / 15$ & $15 / 15$ & NA & $1 / 15$ & $1 / 15$ & $\begin{array}{l}1 / 15 \\
0 / 15\end{array}$ & $\begin{array}{l}6 / 15 \\
0 / 15\end{array}$ & $2 / 15$ & $2 / 15$ & $\begin{array}{l}4 / 15 \\
0 / 15\end{array}$ & NA & NA & NA \\
\hline
\end{tabular}

$\beta$ cat: $\beta$-Catenin; CgA: chromogranin A; CK: cytokeratin; CRT: calretinin; Ecad: E-cadherin; EMA: epithelial membrane antigen; ER: estrogen receptor; INH: inhibin- $\alpha$; NA: not applicable; PR: progesterone receptor; SMA: smooth muscle actin; SNP: synaptophysin; VIM: vimentin; WT1: Wilms tumor 1.

immunohistochemical findings of all 34 MCST cases. In addition to diffuse, strong nuclear $\beta$-catenin immunoreactivity, MCST was characterized by positive expression of sex-cord stromal markers (WT1, forkhead box L2 [FOXL2], and steroid factor 1), indicating a stromal origin. The stromal nature of MCST cells was also suggested by ultrastructural features exhibiting a lack of cell junctions, true luminal space, cilia, microvilli or Reinke crystalloid (3). In the Irving et al. study (11), all cases were positive for FOXL2, suggesting a potential role of FOXL2 immunostaining to distinguish MCST from other ovarian sex cord-stromal tumors. The majority of MCST cases showed no reaction to inhibin- $\alpha$, calretinin or CD56 staining. In addition to distinctive morphological features, immunohistochemical findings of MCST can help discriminate MCST from other sex cord-stromal tumors showing positive expression for inhibin- $\alpha$ and calretinin, such as thecoma, adult granulosa cell tumor, sclerosing stromal tumor and Sertoli-Leydig cell tumor (9). MCST also frequently expressed vimentin, CD10, and cyclin D1, but rarely expressed neuroendocrine markers (synaptophysin and chromogranin A), hormone receptors (estrogen receptor, progesterone receptor and androgen receptor) or epithelial markers (cytokeratin and epithelial membrane antigen). Ki-67 labeling index data were available in $9(26.5 \%)$ cases and ranged from 1 to $16 \%$.

We demonstrated that pyrosequencing can be used to accurately identify point mutations (c.122C $>\mathrm{T}$; p.T41I) in CTNNB1 exon 3 in formalin-fixed, paraffin-embedded tissue sections of ovarian MCST. This finding is consistent with our previous data demonstrating a point mutation (c.98C $>\mathrm{G}$; p.S33C) in two cases of MCST (12) using pyrosequencing. Pyrosequencing is able to detect individual bases or short stretches of nucleic acid sequences at predetermined positions. The technique is a simple, fast and sensitive method that is also cost-effective $(20,21)$. The utility of pyrosequencing may be limited when long stretches of DNA are sequenced. Because of this limitation, as in alternative sequencing methods, prior knowledge of the mutation of interest is required for the clinical application of pyrosequencing (10). As CTNNB1 mutations associated with MCST are frequently found at specific locations, pyrosequencing analysis may be considered a rapid diagnostic tool.

In summary, we described a novel CTNNB1 deletion mutation in ovarian MCST. We also confirmed that a 
previously reported point-mutation of $C T N N B 1$ in ovarian MCST can be accurately identified by pyrosequencing. In addition, we provided a thorough review of previously published cases alongside our own cases and clarified the clinicopathological characteristics of ovarian MCST. Comprehensive analysis of these cases would expand our knowledge regarding ovarian MCST.

\section{Acknowledgements}

This research was supported by Basic Science Research Program through the National Research Foundation of Korea (NRF) funded by the Ministry of Education (2016R1D1A1B03935584).

\section{References}

1 Irving JA and Young RH: Microcystic stromal tumor of the ovary: report of 16 cases of a hitherto uncharacterized distinctive ovarian neoplasm. Am J Surg Pathol 33: 367-375, 2009.

2 Maeda D, Shibahara J, Sakuma T, Isobe M, Teshima S, Mori M, Oda K, Nakagawa S, Taketani Y, Ishikawa S, and Fukayama M: beta-catenin (CTNNB1) S33C mutation in ovarian microcystic stromal tumors. Am J Surg Pathol 35: 1429-1440, 2011.

3 Yang $\mathrm{M}$ and Bhattacharjee MB: Ovarian microcystic stromal tumor: report of a new entity with immunohistochemical and ultrastructural studies. Ultrastruct Pathol 38: 261-267, 2014.

4 Lee SH, Koh YW, Roh HJ, Cha HJ, and Kwon YS: Ovarian microcystic stromal tumor: A novel extracolonic tumor in familial adenomatous polyposis. Genes Chromosomes Cancer 54: 353-360, 2015.

5 Bi R, Bai QM, Yang F, Wu LJ, Cheng YF, Shen XX, Cai X, Zhou XY, and Yang WT: Microcystic stromal tumour of the ovary: frequent mutations of beta-catenin (CTNNB1) in six cases. Histopathology 67: 872-879, 2015.

6 Chen Q, Lu W, and Lv W: Overlap of microcystic stromal tumor and primary solid pseudopapillary neoplasm of the ovary. Int $\mathbf{J}$ Clin Exp Pathol 8: 11792-11797, 2015.

7 Gunes P, Kir G, Yilmaz I, and Kucukodaci Z: Coexistence of Microcystic Stromal Tumor of the Ovary With Mutation of betaCatenin and Contralateral Mucinous Cystadenoma. Int J Gynecol Pathol 34: 546-550, 2015.

8 Podduturi V, Tran T, Champion KJ, Onur N, and Shiller SM: Microcystic Stromal Tumor of the Ovary: A Case Report of a Newly Described Ovarian Neoplasm With a beta-catenin (CTNNB1) G34E Mutation. Int J Gynecol Pathol 34: 541-545, 2015.

9 Kang YN, Cho CH, and Kwon SY: Microcystic stromal tumor of the ovary with mutation in exon 3 of beta-catenin: a case report. Int J Gynecol Pathol 34: 121-125, 2015.

10 Liu C, Gallagher RL, Price GR, Bolton E, Joy C, Harraway J, Venter DJ, and Armes JE: Ovarian Microcystic Stromal Tumor: A Rare Clinical Manifestation of Familial Adenomatous Polyposis. Int J Gynecol Pathol 35: 561-565, 2016.
11 Irving JA, Lee CH, Yip S, Oliva E, McCluggage WG, and Young RH: Microcystic Stromal Tumor: A Distinctive Ovarian Sex Cord-Stromal Neoplasm Characterized by FOXL2, SF-1, WT-1, Cyclin D1, and beta-catenin Nuclear Expression and CTNNB1 Mutations. Am J Surg Pathol 39: 1420-1426, 2015.

12 Lee JH, Kim HS, Cho NH, Lee JY, Kim S, Kim SW, Kim YT, and Nam EJ: Genetic analysis of ovarian microcystic stromal tumor. Obstet Gynecol Sci 59: 157-162, 2016.

13 Buda A and Pignatelli M: E-cadherin and the cytoskeletal network in colorectal cancer development and metastasis. Cell Commun Adhes 18: 133-143, 2011.

14 Mulligan WA, Wegner KA, Keil KP, Mehta V, Taketo MM, and Vezina CM: Beta-catenin and estrogen signaling collaborate to drive cyclin D1 expression in developing mouse prostate. Differentiation 93: 66-71, 2017.

15 Shi L, Wu YX, Yu JH, Chen X, Luo XJ, and Yin YR: Research of the relationship between beta-catenin and c-myc-mediated Wnt pathway and laterally spreading tumors occurrence. Eur Rev Med Pharmacol Sci 21: 252-257, 2017.

16 Bahrami A, Amerizadeh F, ShahidSales S, Khazaei M, GhayourMobarhan M, Sadeghnia HR, Maftouh M, Hassanian SM, and Avan A: Therapeutic Potential of Targeting Wnt/beta-catenin Pathway in Treatment of Colorectal Cancer: Rational and Progress. J Cell Biochem, 2017. doi: 10.1002/jcb.25903. [Epub ahead of print]

17 Takayasu H, Horie H, Hiyama E, Matsunaga T, Hayashi Y, Watanabe Y, Suita S, Kaneko M, Sasaki F, Hashizume K, Ozaki T, Furuuchi K, Tada M, Ohnuma N, and Nakagawara A: Frequent deletions and mutations of the beta-catenin gene are associated with overexpression of cyclin D1 and fibronectin and poorly differentiated histology in childhood hepatoblastoma. Clin Cancer Res 7: 901-908, 2001.

18 Pineau P, Marchio A, Battiston C, Cordina E, Russo A, Terris B, Qin LX, Turlin B, Tang ZY, Mazzaferro V, and Dejean A: Chromosome instability in human hepatocellular carcinoma depends on p53 status and aflatoxin exposure. Mutat Res 653: 6-13, 2008.

19 Farkkila A, McConechy MK, Yang W, Talhouk A, Ng Y, Lum A, Morin RD, Bushell K, Riska A, McAlpine JN, Gilks CB, Unkila-Kallio L, Anttonen M, and Huntsman DG: FOXL2 402C $>$ G Mutation Can Be Identified in the Circulating Tumor DNA of Patients with Adult-Type Granulosa Cell Tumor. J Mol Diagn 19: 126-136, 2017.

20 How-Kit A and Tost J: Pyrosequencing(R)-Based Identification of Low-Frequency Mutations Enriched Through Enhanced-iceCOLD-PCR. Methods Mol Biol 1315: 83-101, 2015.

$21 \mathrm{Kim}$ HJ, Oh SY, Kim WS, Kim SJ, Yoo GH, Kim WD and Lee $\mathrm{KY}$ : Clinical investigation of EGFR mutation detection by pyrosequencing in lung cancer patients. Oncol Lett 5: 271-276, 2013.

Received March 16, 2017

Revised April 14, 2017

Accepted April 18, 2017 